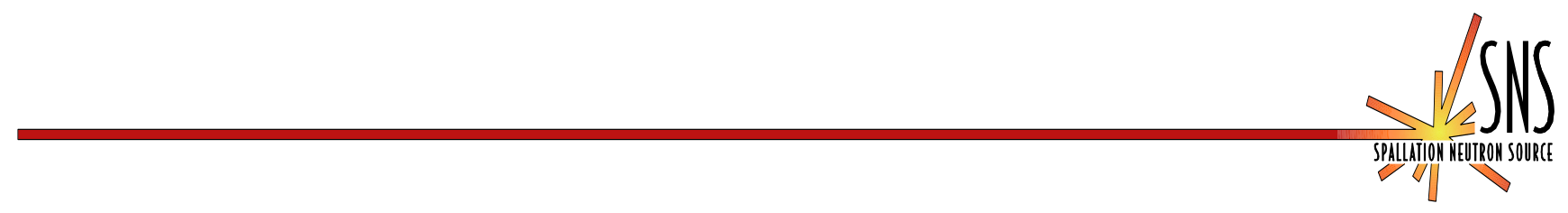

\title{
SNS Extraction Kicker System Impedance Estimate
}

\author{
BNL/SNS Technical Note
}

No. 140

H. Hahn

November 10, 2004

Collider-Accelerator Department Brookhaven National Laboratory

Upton, New York \#11973 


\title{
SNS Extraction Kicker System Impedance Estimate
}

\author{
H. Hahn
}

\section{Introduction}

The recent availability of production SNS extraction kickers, three large-aperture magnets and one small- aperture magnet suggests to review and update the transverse impedance estimates for use in the beam stability analysis. The first estimate was presented by Davino. Mi, and Tsoupas ${ }^{1}$ and was based on the full- and half size prototype magnet. An updated estimate ${ }^{2}$ became possible after the completion of the impedance measurements on the small-aperture magnet, K11. New results from three large-aperture magnets, (K25, K26, K27), will here be combined with those from the prototype (PT) and K11 for a final estimate before start of operation. In view of measuring errors, both systematic and random, the system impedance estimate is given as a best guess for the lower limit with a range to the upper limit.

The impetus for the latest measurements was the need to evaluate the effect of TiN coating on the magnet impedance. The TiN coated SNS extraction kicker magnet, K26, together with two uncoated magnets was assembled in the down-stream vacuum vessel. In this magnet, a $0.1 \mu \mathrm{m}$ thick TiN coating is applied to reduce secondary electron emission from ferrite blocks in order to suppress electron multipacting. The coating is applied in the form of longitudinal stripes, spaced $1.5 \mathrm{~cm}$ apart and electrically isolated from each other and the busbar. Transverse impedance measurements were performed which showed no difference between the magnets. Details of the measuring procedure and results are presented in this note. High power testing of the magnet has been done confirming that TiN coating of all magnets is a permissible treatment. ${ }^{3}$

\section{Impedance Measurements of the TiN coated magnet}

The three large-aperture magnets $\left(\mathrm{K}^{* *}\right)$ are installed in the production down-stream vacuum vessel in their proper location and with eddy current strips connected. The magnets have the same geometrical dimensions of $h=24.3 \mathrm{~cm}$ in gap height, $w=15.1$ $\mathrm{cm}$ in width, and $\ell=39 \mathrm{~cm}$ in length. Their geometry is similar to the prototype magnet 
with corresponding $24.3 \mathrm{~cm}, 15.9 \mathrm{~cm}$ and $36 \mathrm{~cm}$ dimensions, allowing a straight forward comparison with previous measurements.

The magnets in the $3.78 \mathrm{~m}$ long vessel are only accessible to electrical measurements directly at the busbar, free standing or connected with the feedthrough to the PFN. Consequently, standard twin-wire measurements are not feasible and the coupling impedance is determined by the "direct method". ${ }^{4}$ The direct method gives the "coupled" termination-dependent impedance correctly but can not yield the intrinsic image current induced impedance. At low frequencies, below $<10 \mathrm{MHz}$, the ferrites have low losses and the intrinsic impedance is negligible compared to those from the $25 \Omega$ termination. If necessary at higher frequencies, one can add the appropriate values from the prototype.

The input impedance at the busbar of the bare magnets, without feedthrough and disconnected from the PFN, is shown in Fig. 1. The measured inductance is $L_{B}=1.02 \mu \mathrm{H}$ and the corrected magnet inductance is $L_{K} \approx 0.96 \mu \mathrm{H}$ after correction for the clip lead inductance. The estimated inductance is between $\mu_{0} h \ell / w=0.789$ and $\mu_{0} h(\ell+w) / w=$ $1.094 \mu \mathrm{H}$. Also shown in Fig. 1 is the input impedance of the magnets with the feedthrough but still without the PFN. The feedthrough adds capacitance at the busbar plates thereby lowering the resonance frequency. It is important to note that all three magnets show the same pattern, thus proving that TiN coating has no negative impact on the coupling impedance.
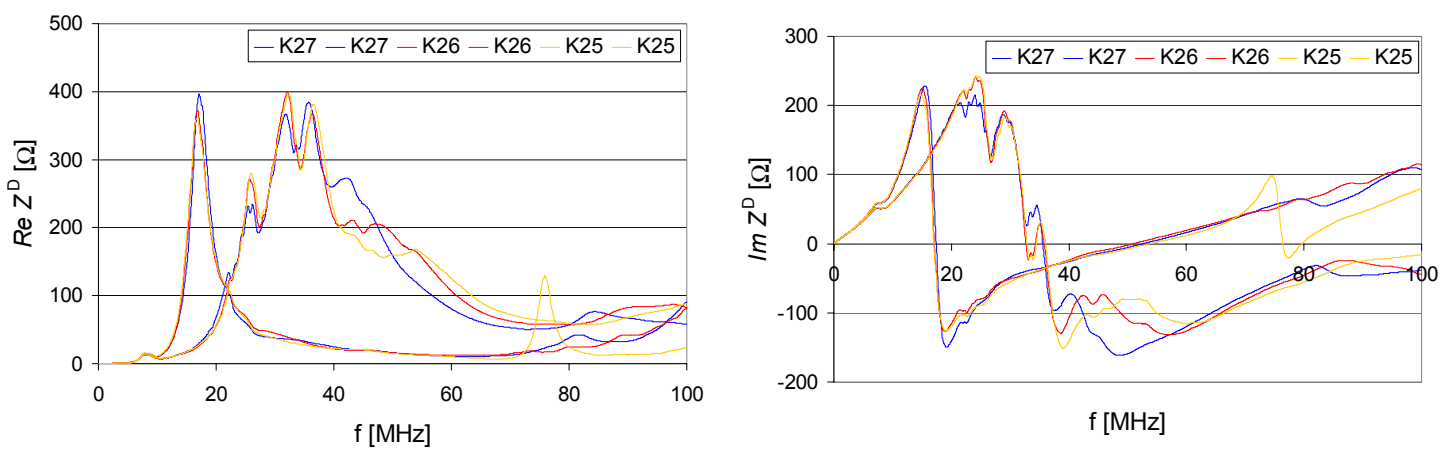

Fig. 1. Impedance at busbar of TiN coated magnet without PFN

The kicker magnet is located in a vacuum vessel with feedthrough and is electrically connected to the Pulse Forming Network (PFN) by means of two $\sim 200 \mathrm{~m}$ long $50 \Omega$ cables. The PFN is designed for a $25 \Omega$ output impedance. The cables are in parallel to 
match to the $25 \Omega$ magnet termination. The input impedance with the PFN connected to each of the magnet is shown in Fig. 2. The mismatch of the PFN appears in the impedance as rapid oscillation, with the frequency defined by the cable length.

Considerable efforts had been made to minimize the mismatch, such as screening the PFN load resistor and separating the grounding of the magnet vessel from the PFN ground. Adding an RC shunt at the PFN terminal was considered, but left as a possible addition if required by operation. The prototype magnet impedance with and without the $25 \Omega$ plus $100 \mathrm{pF}$ RC shunt is shown in Fig.3, pointing to no obvious preference.
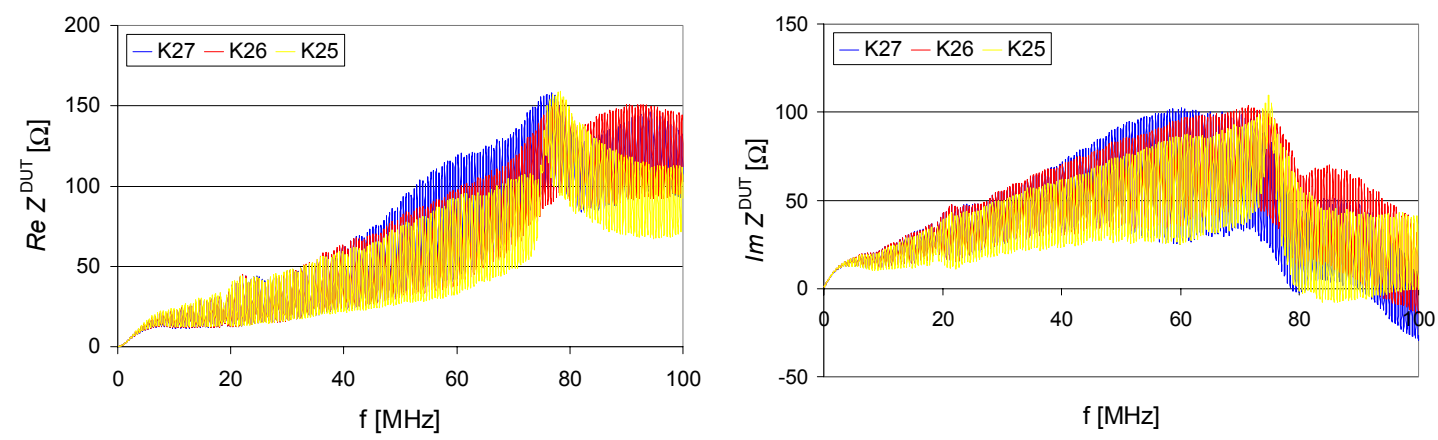

FIG. 2. Impedance at busbar for $\mathrm{K}^{* *}$ magnets with PFN termination.
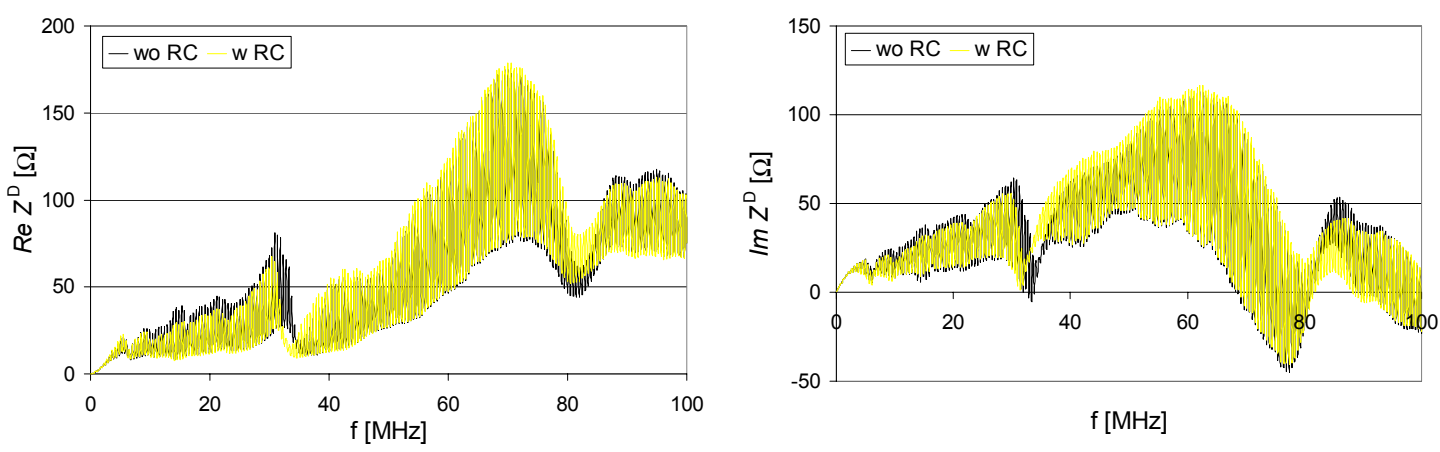

Fig. 3. Impedance at busbar of prototype magnet for PFN with and without RC shunt.

\section{Vertical impedance of the large-aperture magnets, K25, 26, 27}

The direct measurement of the impedance at the busbar yields the vertical impedance by taking

$$
Z_{y}=\frac{c}{\omega h^{2}} Z^{D}
$$


with $h=24.3$ the gap height. A more detailed analysis of the direct method suggest that the results may be pessimistic, that is the measured impedance is $\sim 15 \%$ high.

Furthermore, the image induced intrinsic impedance is ignored by the direct method. The intrinsic impedance is due to ferrite losses and thus at the low frequencies in Fig. 4 has been neglected.
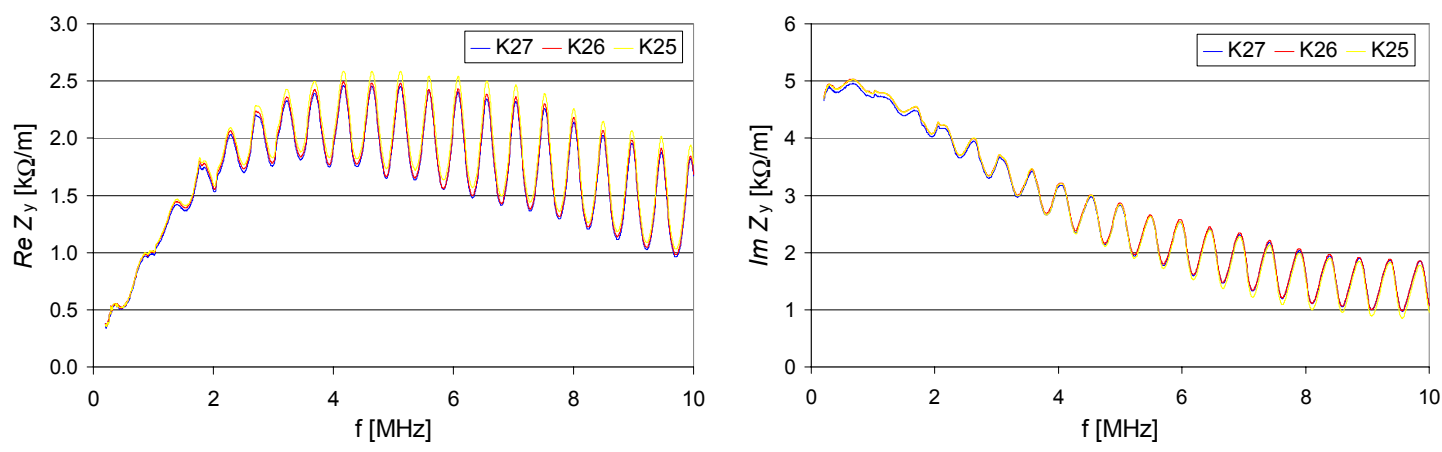

Fig. 4. Vertical impedance of the kicker magnets K25, K26, K27.

The vertical coupled impedance at higher frequencies is shown in Fig. 5, together with the intrinsic impedance from the prototype magnet. At higher frequencies, $>10 \mathrm{MHz}$, the real part of intrinsic impedance is noticeable and the twin-wire result for the prototype magnet can be added as $Z_{I}^{D} \approx \omega \times 0.003 \mu \mathrm{H}$ below $50 \mathrm{MHz}$ or as $Z_{y I} \approx 0.5 \pm 0.4 \mathrm{k} \Omega / \mathrm{m}$ depending on frequency. The imaginary contribution of the intrinsic impedance is small enough to be neglected at all frequencies. It must be noted that the results for the intrinsic contribution are the least reliable. The three magnets are quite similar and the impedance is best presented by taken their average value in Fig. 5 .
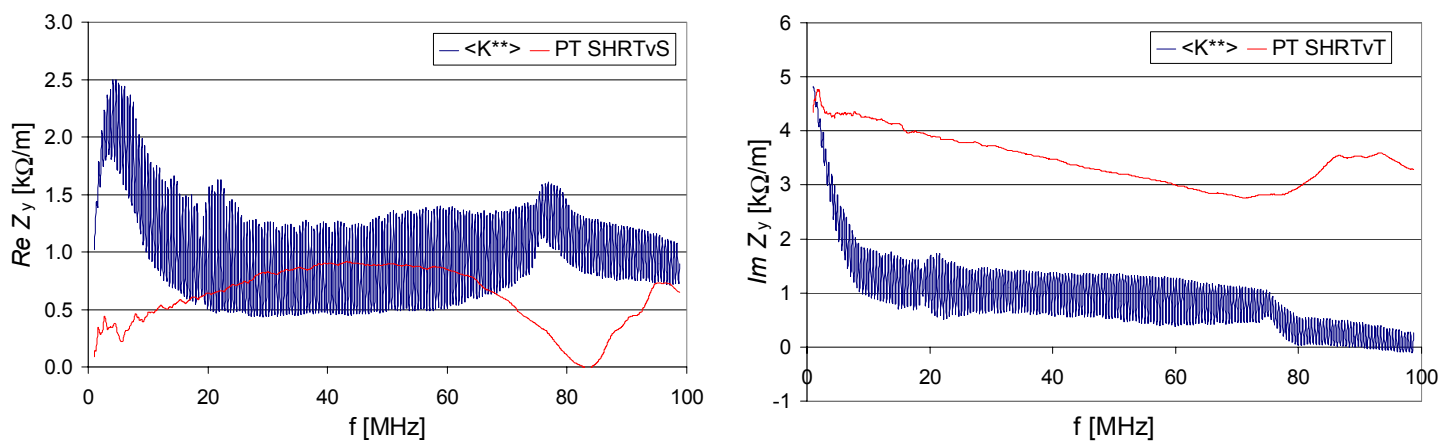

FIG.5. Vertical Impedance of one large-aperture $\mathrm{K}^{* *}$ kicker magnet 
The geometry of the large aperture production magnets is close to that of the prototype. The gap height is $24.3 \mathrm{~cm}$ in both magnets, the width are 15.1 versus $15.9 \mathrm{~cm}$, and the lengths are 39 versus $36 \mathrm{~cm}$ in the production and prototype magnets respectively. The vertical impedance of the prototype magnet is shown in Fig. 6 for comparison. The imaginary impedance value (SHRTvT, red) in Fig. 6 is obtained from the shorted busbar with a twin wire measurement versus a reference tube and is due to the leakage inductance and thus is not seen by the beam.
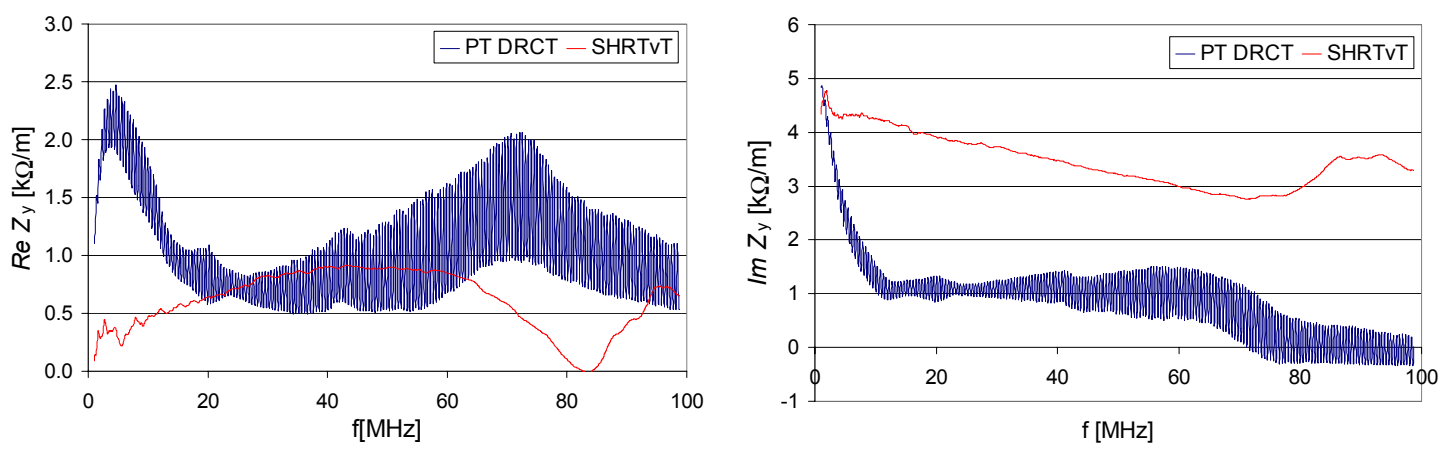

FIG. 6. Vertical Impedance of the prototype kicker magnet

\section{Vertical impedance of the small-aperture magnet K11}

Comparing the large-aperture magnet with the smallest aperture magnet K11 advances the general understanding of the kicker magnet properties and is helpful for an updated coupling impedance estimate of the total SNS extraction system. The geometric dimensions of the small-aperture magnet are a gap of $16.6 \mathrm{~cm}$, a width of $12 \mathrm{~cm}$, and a length of $40 \mathrm{~cm}$. The vertical impedance, both coupled and uncoupled, of the PFN terminated K11 magnet is shown in Fig. 7. Since the $\mathrm{K}^{* *}$ magnets had to be measured with the direct method, only the corresponding data for K11 are used here for the total system estimate. 

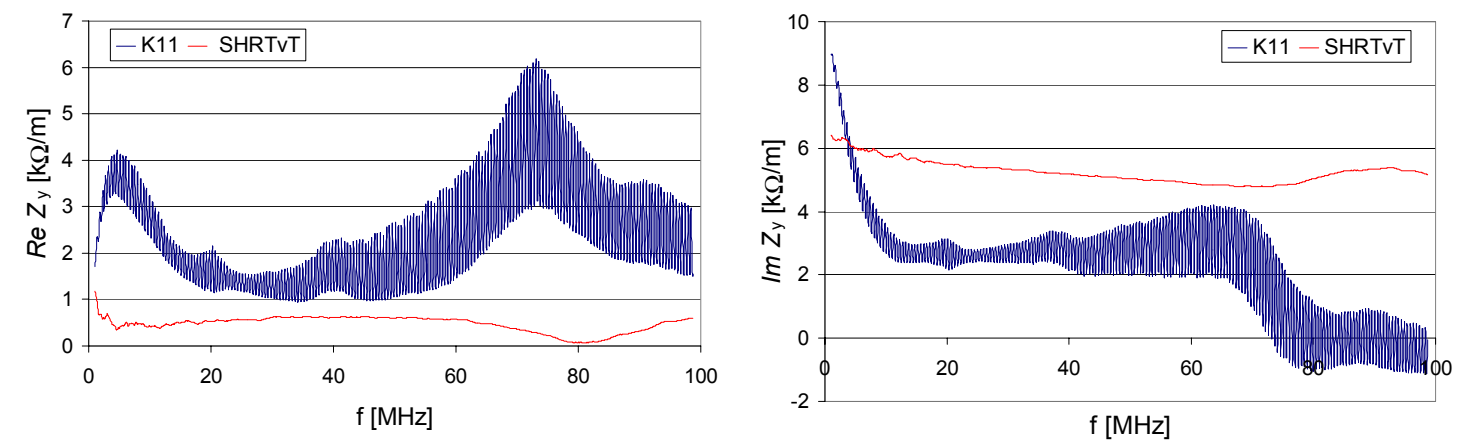

FIG. 7. Vertical Impedance of the PFN terminated K11 kicker magnet

\section{Scaling of Kicker Magnet Impedance}

The availability of the production magnets $\mathrm{K} 11$ and K25, K26, K27 provided the opportunity for a review of the scaling laws used in the generation of an impedance estimate for the extraction kicker magnet system.

The vertical impedance of a kicker magnet connected to the external load, here the PFN, has two principal contributions, the impedance coupled to the termination, $Z_{g}$, and the intrinsic impedance, here $R_{I}$, each of which has to be scaled separately. Although some details have been neglected, the equivalent circuit in Fig. 8 serves adequately for the interpretation of the available magnet data.

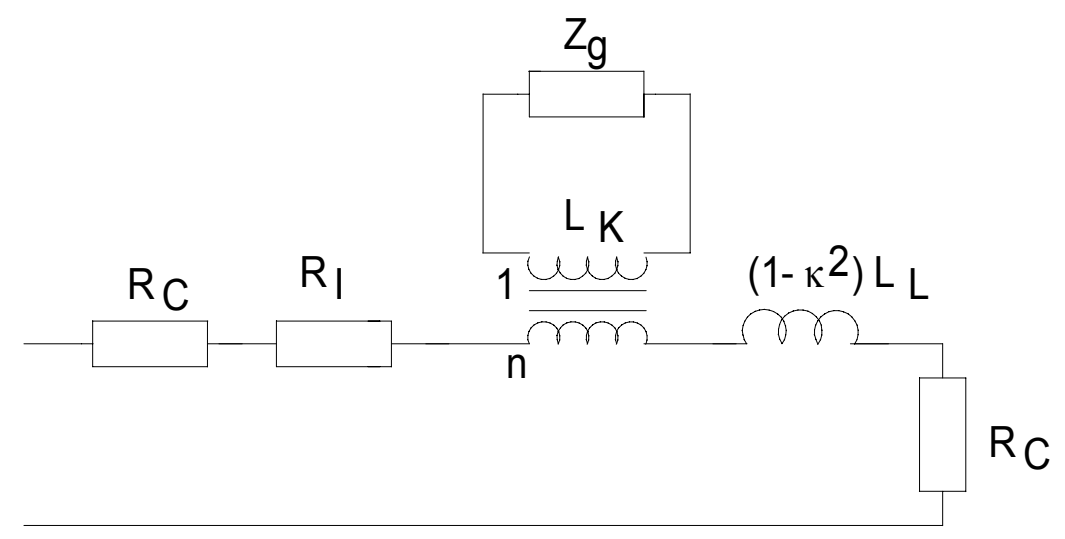

FIG. 8. Equivalent circuit for impedance measurements

The coupled impedance is determined by the PFN termination, $Z_{g}$. It is well represented by the resonant model with the magnet inductance $L_{K}$, the busbar 
capacitance, $C_{B}$, and the termination, $Z_{g}$ in parallel. The coupled impedance can be measured by the twin-wire method versus short or the direct method at the busbar. The vertical impedance follows from the measured input impedance at the busbar according to $Z_{y}=Z^{D} c /\left(\omega h^{2}\right)$ with $h$ the gap height between the busbar.

The model predicts quadratic scaling ${ }^{4}$ and is used in ref. 1 . Scaling can be considered to be a magnet property independent of the termination. Comparable date for all magnets, $\mathrm{K}^{* *}, \mathrm{~K} 11$, and Prototype are only available from the direct method and will be used here to confirm the quadratic scaling law. In order to obtain clean signals for the vertical impedance, the K11 and production magnets were terminated with a local $25 \Omega$ resistor and the results are shown in Fig. 9. Quadratic scaling leads to a ratio of $(24.3 / 16.6)^{2}=2.14$, in remarkable agreement with the data.
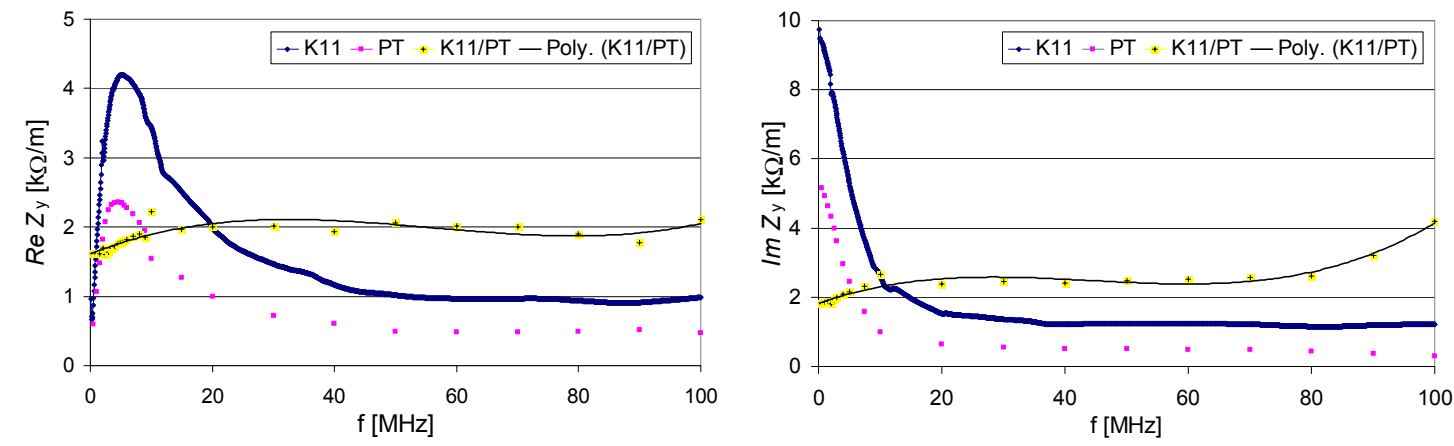

FIG. 9. Coupled impedance ratio of K11 and prototype

The intrinsic impedance $Z_{y I}=R_{I} c /\left(\omega \Delta^{2}\right)$ is image-current generated and must be measured with the twin-wire method on the magnet with shorted busbar. The intrinsic impedance is due to ferrite losses, it is almost pure resistive but frequency dependent. Typically, this impedance is small compared to the coupled impedance and can be ignored at low frequencies, below $\sim 10 \mathrm{MHz}$. However, at higher frequencies the external termination part in the coupled impedance is shunted by the busbar capacitance, so that the intrinsic impedance relatively growths and can become a significant contribution to the resistive impedance part. In ref. 1, the scaling of the intrinsic impedance is conjectured to follow quadratically with the gap width, perpendicular to the kick direction, but this could not be confirmed here by the measurements. Results from wire 
measurements on the shorted K11 and prototype magnets are shown in Fig. 9. For the purpose of system scaling, no contribution will be made below $1 \mathrm{MHz}$, and the average value $(<\mathrm{PT}, \mathrm{K} 11>$,black) will be used at higher frequencies for all magnets.

The twin wire measurement adds a misleading reactive term due to the leakage inductance, $\kappa^{2} L_{L}$ which is not image current induced and thus is not seen by the beam. It follows that no correction to the direct measured results is required for the imaginary impedance. Assuming the first order approximation for $\kappa^{2} L_{L} \approx(\Delta / h)^{2} L_{K}$ and $L_{K} \approx \mu_{0}(h / w) \ell$ leads to the scaling according to , $Z_{y I} \approx \ell /(h w)$ or applied here to the theoretical impedance ratio of $\sim 1.9$, consistent with the measured $\sim 1.5$ in Fig. 10.
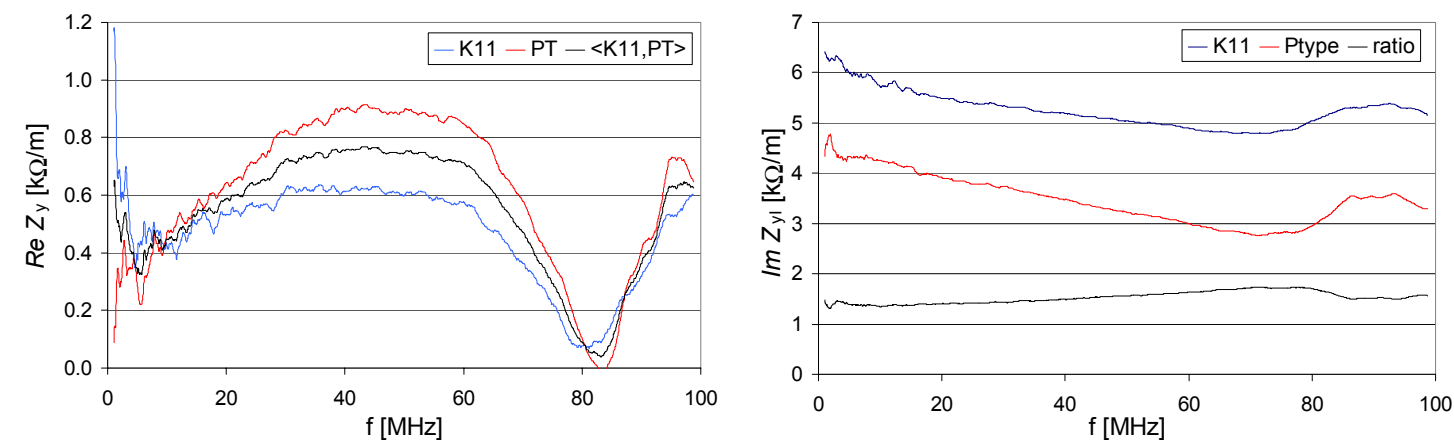

FIG. 10. Uncoupled impedances of K11 and prototype magnets.

\section{Updated Impedance estimate for the SNS Extraction kicker system}

Estimates for the transverse coupling impedance of the SNS Extraction Kicker system were previously provided by Davino et al. ${ }^{1}$ The estimates were based on a model in which the coupled and uncoupled impedances are represented by simple resonate circuits. The contributions from the 14 kicker magnets were obtained by quadratically scaling with respect to the magnet gap height and width for the coupled and the width for the uncoupled impedance respectively and by summing the individual contributions. Scaling of the magnet gap height while maintaining the impedance was used to minimize the total impedance. The magnet gap width, height, and length of the 14 magnets is listed below, and quadratic scaling factors with respect to height and the combination of height and length are given here. 


\begin{tabular}{|c|c|c|c|c|c|c|c|}
\hline$\overline{\mathrm{K} 11}$ & 12 & 16.6 & 40 & 1.46 & 2.2 & 1 & 1 \\
\hline $\mathrm{K} 12$ & 14.5 & 20 & 40 & 1.22 & 1.51 & 0.83 & 0.69 \\
\hline K13 & 14.5 & 20 & 40 & 1.22 & 1.51 & 0.83 & 0.69 \\
\hline K14 & 17.8 & 19.5 & 51 & 1.25 & 2.03 & 0.85 & 0.92 \\
\hline K15 & 17.8 & 19.5 & 51 & 1.25 & 2.03 & 0.85 & 0.92 \\
\hline K16 & 21.1 & 23.3 & 51 & 1.04 & 1.42 & 0.71 & 0.65 \\
\hline K17 & 21.1 & 23.3 & 51 & 1.04 & 1.42 & 0.71 & 0.65 \\
\hline K21 & 16.2 & 23.3 & 43 & 1.04 & 1.2 & 0.71 & 0.55 \\
\hline K22 & 16.2 & 23.3 & 43 & 1.04 & 1.2 & 0.71 & 0.55 \\
\hline K23 & 16.2 & 23.3 & 43 & 1.04 & 1.2 & 0.71 & 0.55 \\
\hline K24 & 16.2 & 23.3 & 43 & 1.04 & 1.2 & 0.71 & 0.55 \\
\hline K25 & 15.1 & 24.3 & 39 & 1 & 1 & 0.68 & 0.45 \\
\hline K26 & 15.1 & 24.3 & 39 & 1 & 1 & 0.68 & 0.45 \\
\hline \multirow[t]{2}{*}{ K27 } & 15.1 & 24.3 & 39 & 1 & 1 & 0.68 & 0.45 \\
\hline & & & & $\overline{17 .}$ & 19.93 & 8.27 & 9.07 \\
\hline
\end{tabular}

The entry for the extraction system vertical impedance in the SNS impedance budget was summarized in ref. 1 as $\operatorname{Re} Z_{y} \approx 33.2 \mathrm{k} \Omega / \mathrm{m}$ for a frequency below $10 \mathrm{MHz}$ and $\operatorname{Re} Z_{y} \approx 12 \mathrm{k} \Omega / \mathrm{m}$ for frequencies at $\sim 50 \mathrm{MHz}$.

As discussed above, the new information from the four production magnets permits to form an updated estimate of the total extraction kicker system. This estimate gives equal weight to the large and small aperture magnet results. The present estimate for the coupled part assumes quadratic scaling with gap height and linear scaling with magnet length. Based on the detailed dimensions of the magnets in the above Table, and summing the scaled contribution from each magnet leads to the expression

$$
Z_{y}=\frac{1}{2}\left[19.93 \times Z_{y}\left(K^{* *}\right)+9.07 \times Z_{y}(K 11)\right]
$$

Results are available for the three kicker-PFN combinations only from direct measurements so that the estimate has been based on those comparable for the production magnets, $\mathrm{K}^{* *}$ and $\mathrm{K} 11$. The intrinsic impedance is taken as 14 times the average value from K11 and the production magnet in Fig. 10.

The updated system impedance is now shown in Fig. 11. One curve for the real impedance part, $\left(Z_{y}+Z_{y} I\right.$, blue $)$ represents the scaled sum of coupled plus the intrinsic impedance. This curve can be considered as upper limit of the impedance estimate. The second curve $\left(0.85 Z_{y}\right.$, red $)$ is the coupled impedance with a small correction factor for 
the stray inductance of the busbar straps. ${ }^{5}$ The best guess of the lower limit for the real part is represented by the black curve, $\left\langle Z_{y}\right\rangle$, which is obtained by averaging the blue and red curve and smoothing over $1.2 \mathrm{MHz}$ to take out the oscillations. The best guess value for the peak at $\sim 4.5 \mathrm{MHz}$ is $40(+15 \%) \mathrm{k} \Omega / \mathrm{m}$ and for the secondary peak at $\sim 75$ $\mathrm{MHz}$ is $34(+15 \%) \mathrm{k} \Omega / \mathrm{m}$. The same rules were applied to the imaginary impedance part with the assumption that the intrinsic impedance is primarily resistive.
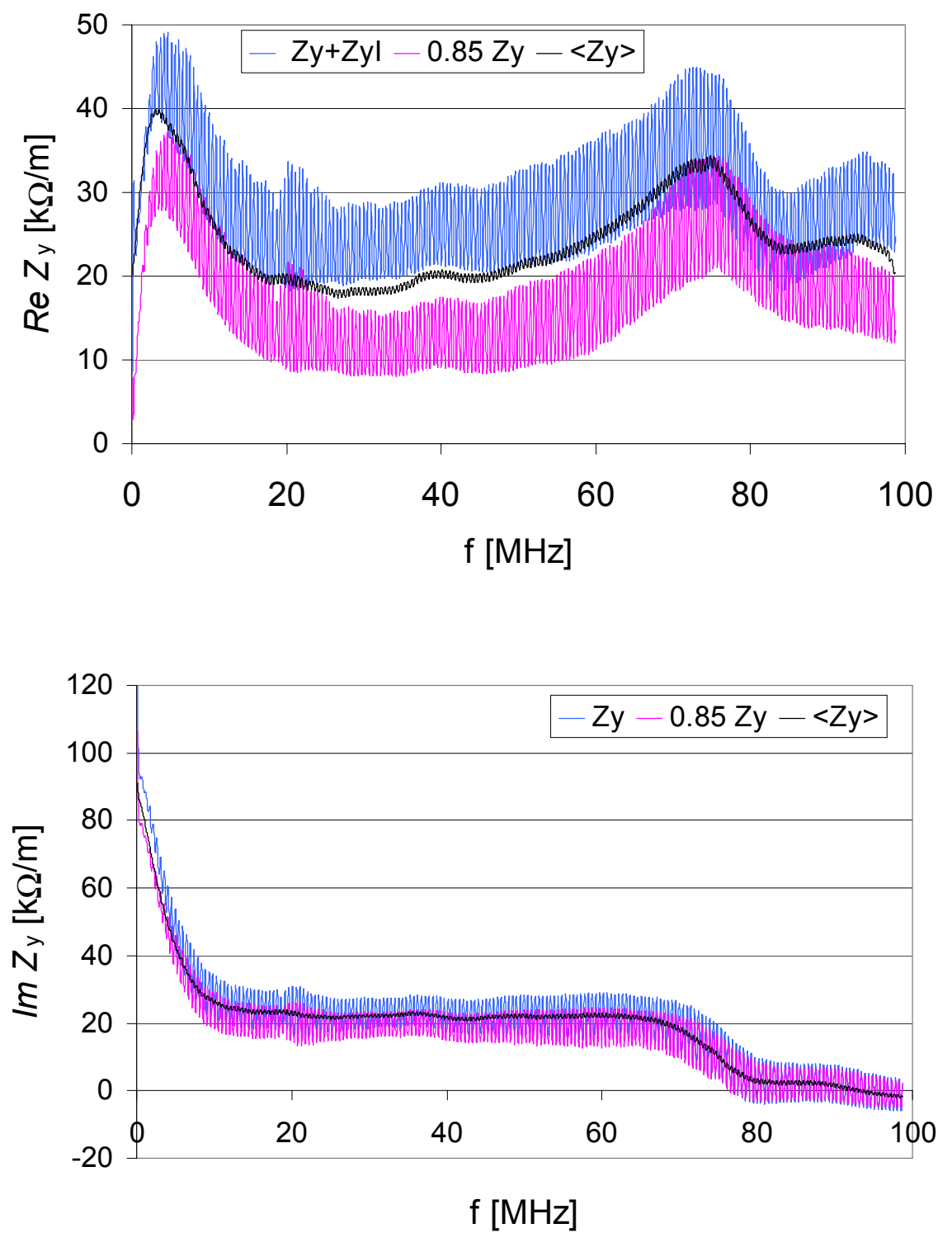

Fig. 11. Coupling impedance estimate for the SNS extraction kicker system 
1. D.Davino, J. Mi, N. Tsoupas, BNL/SNS TN \#112 Rev. 1 (Aug. 2002)

2. H. Hahn, BNL/SNS TN \# 135 (May 2004)

3. J.-L. Mi and D. Warburton, private communication

4. D. Davino and H. Hahn, Phys. Rev. ST Accel. Beams, 6, 012001 (2003)

5. H. Hahn, Phys. Rev. ST Accel. Beams, (in print) 